\title{
CT in every step: Long COVID
}

\author{
Sonay Aydin ${ }^{1}$, Edhem Unver $^{1}$, Erdal Karavas ${ }^{1}$, Seven Yalcin $^{1}$, and Mecit Kantarci ${ }^{2}$ \\ ${ }^{1}$ Erzincan Binali Yildirim University \\ ${ }^{2}$ Ataturk University
}

February 18, 2021

\begin{abstract}
Background and aim: Some patients continue to experience symptoms related to COVID-19 after the acute phase of infection. Imaging studies, especially chest computerized tomography (CT), has been gaining importance from the beginning of the pandemic with its ability of diagnosing COVID-19, assessing the extent of pulmonary involvement, predicting disease severity. We intend to define the frequency of persistent symptoms and correlate the presence of persistent symptoms with laboratory findings and CT severity levels. Methods and Materials: We tried to patients who had been discharged from the study hospital after the treatment and who had a positive nasopharyngeal swab result for SARS-CoV-2, after at least four weeks from the initial diagnosis. The patients were questioned for the presence of persisting symptoms. In addition to demographic data, laboratory results and CT severity levels were recorded. Results: 116 patients were included into the study. 61 patients reported at least one persisting symptom (52.5\%). Mean age of the population is $48.90 \pm 17.74$ years. Shortness of breath, chest pain, cough, muscle weakness, dizziness, headache, fatigue, and palpitation were found as persisting symptoms. Mean CT severity score value of the population is $3.80 \pm 0.38$. Mean CT severity score value is lower in patients without any persistent symptoms. Conclusion: CRP, fibrinogen levels, anemia and female gender were associated with some of the persistent symptoms. CT severity, as being a successful predictor for disease severity/prognosis, is also related with future long COVID presence, and CT severity is related with more persistent symptoms than laboratory parameters.
\end{abstract}

\section{Hosted file}

Manuscript.pdf available at https://authorea.com/users/396460/articles/509627-ct-in-everystep-long-covid

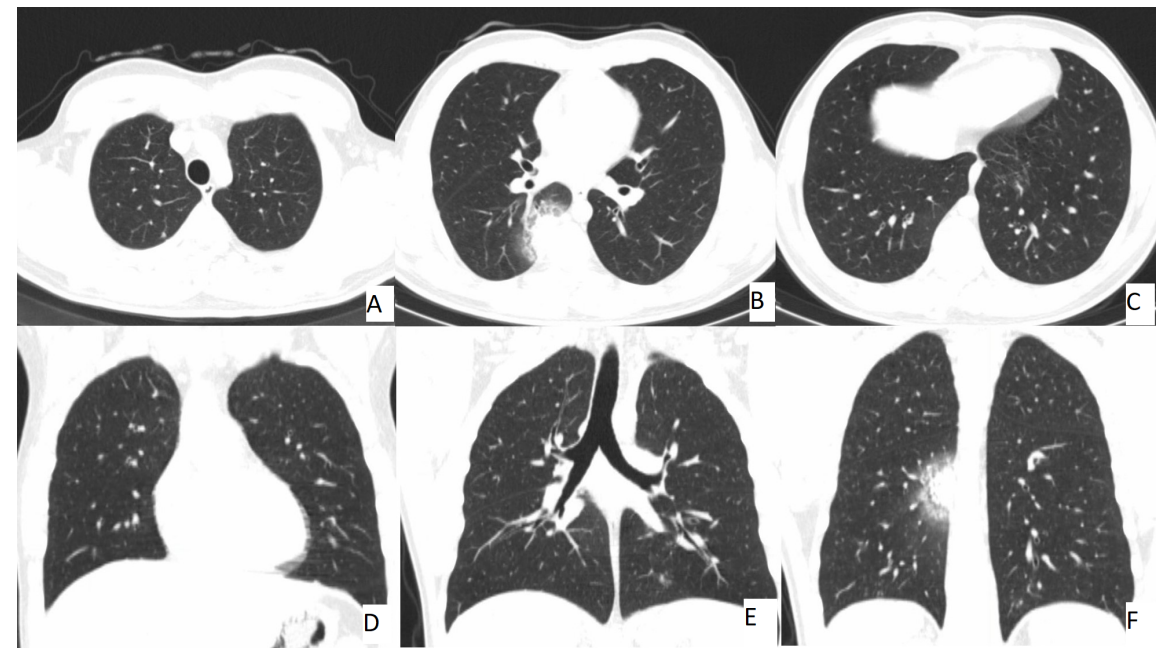




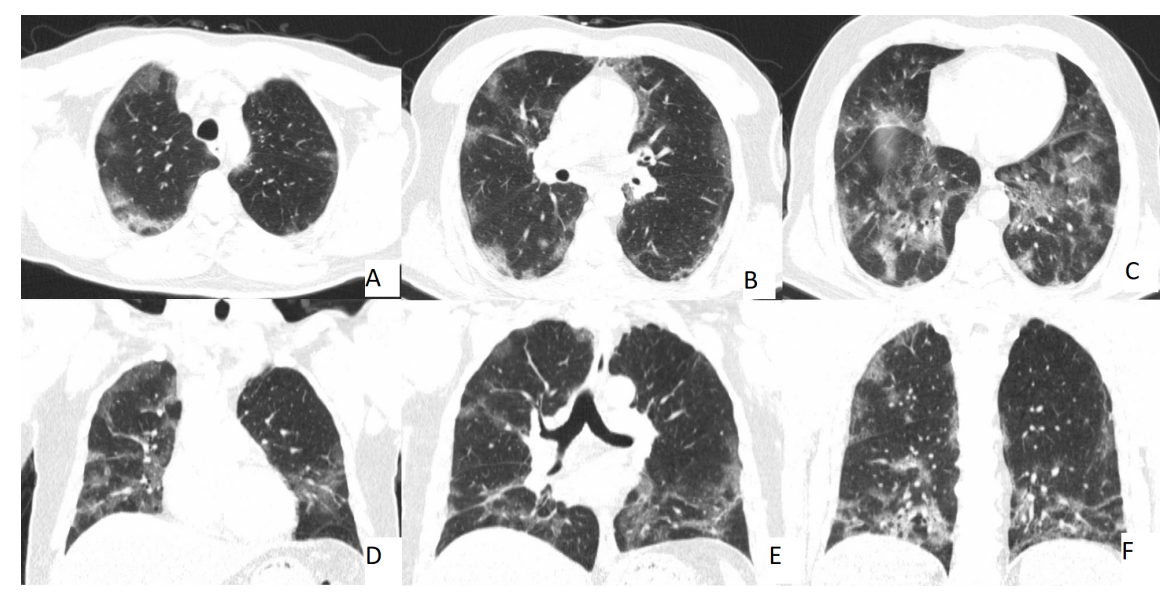

